\title{
¿INCENTIVAN Y PROMUEVEN LOS ESTUDIOS DE POSTGRADO LA INVESTIGACIÓN Y LA PRODUCCIÓN INTELECTUAL?
}

\author{
DO POSTGRADUATE STUDIES INCENTIVE \\ AND PROMOTE RESEARCH AND INTELECTUAL \\ PRODUCTION?
}

\author{
Ingeborg Zenner de Polanía ${ }^{1}$ \\ María Teresa Ríos Tovar ${ }^{2}$
}

\section{RESUMEN}

Para analizar si en para Colombia se cumple la regla establecida por los países desarrollados que los postgrados, las maestrías y específicamente los doctorados obtenidos en cualquier disciplina facultan a los profesionales para realizar investigaciones y lógicamente hacer asequibles los resultados a otros científicos, a nivel nacional e internacional, se analizaron y se compararon los antecedentes disponibles de los líderes de grupos de investigación, correspondientes a cuatro de los once programas nacionales de Ciencia y Tecnología reconocidos y establecidos por el Instituto Colombiano para el Desarrollo de la Ciencia y la Tecnología - COLCIENCIAS hasta el año 2006. Se logró comprobar, de manera contundente que, o la mayoría de los docentes en la educación superior o investigadores de centros especializados no investigan o si lo hacen, no difunden, de manera apropiada, los nuevos conocimientos o tecnologías obtenidos. El género femenino muestra, proporcionalmente, una mayor producción intelectual, a pesar de que sigue en desventaja en cuanto al postgrado, en comparación con el masculino. La divulgación de los

1 I.A., M.Sc., Ph.D. Docente Investigadora, Programa de Ingeniería Agronómica, Universidad de Ciencias Aplicadas y Ambientales U.D.C.A izenner@udca.edu.co

2 Estadística, Coordinadora Área Estadística, Departamento de Planeación, U.D.C.A marrios@udca.edu.co resultados a través de revistas indexadas, nacionales e internacionales, aunque ocupa un porcentaje importante del total es equitativamente similar a los artículos publicados en revistas no indexadas. Finalmente llama la atención el hecho de la mayor producción de las universidades privadas en comparación con las oficiales, a pesar de disponer estas últimas de un número mayor de docentes, líderes de grupos de investigación reconocidos, con títulos de postgrado.

Palabras clave: postgraduados, investigación, visibilidad, género.

\section{SUMMARY}

To analyze, if in Colombia the rule, coined by developed countries, that postgraduate education, masters or specially doctorates obtained within any discipline, empowers professionals to realize research and logically make available the results to other scientists at national and international levels, the available antecedents of the leaders of research groups, corresponding to four of the eleven national programs of Science and Technology, recognized and established by Colciencias up to 2006, were analyzed and compared. It was successfully proven that, either the mayor part of university professors or researchers within specialized centers do not investigate or if they do so, the new knowledge or 
technologies obtained is not diffused appropriately. The female gender shows proportionally a higher intellectual production, even so women continue in disadvantage with regard to postgraduate education compared with men. Result publication within national or international indexed journals, although occupy an important percentage of the total, is equitably similar to articles published in non-indexed serials. Finally attention was attracted by the fact of the mayor production of private universities in comparison with the official ones, in spite of having the public universities at its disposal a higher number of professors, leaders of research groups, with postgraduate titles.

Key words: postgraduates, research, visibility, gender.

\section{INTRODUCCIÓN}

Colombia se ha propuesto pertenecer a la "Sociedad del Saber", la cual se relaciona íntimamente con la "Sociedad de la Información", denominación que recibe la primera década del siglo actual (Zenner de Polanía, 2004). Para alcanzar estos propósitos, se debe disponer de talento humano, capacitado para la transformación de la realidad contemporánea con sus investigaciones, es decir, contar con profesionales, con postgrados, quienes por su educación y su experiencia específica han sido capacitados para afrontar el reto y llevar a cabo la tarea de aumentar los conocimientos en ciencia y en tecnología. Al respecto, Cárdenas (1999) afirma que entre los atributos de la sociedad del saber está la necesidad de preparar más profesionales para realizar estas actividades. Agrega la UNESCO (1999), durante la conferencia mundial sobre ciencia cuyo lema era "la ciencia en el siglo XXI: un nuevo compromiso", que el futuro dependerá cada vez más de una producción equitativa del conocimiento, lo que implica, ente otros retos, la capacidad científica y la participación de la mujer en la ciencia y la tecnología de los países en vía de desarrollo. Agrega Valenti López (2002), quien habla de la "Sociedad del Conocimiento o de la Economía del Conocimiento", que para cumplir con la formación de esta sociedad, las instituciones de los países en vías de desarrollo deben comprender las "determinantes de este fenómeno y poder identificar los mecanismos y variables a considerar en una estrategia hacia la construcción". Entre estos mecanismos, se encuentra la educación avanzada.
Uno de los doctorados en Ciencias Políticas y Sociales de mayor prestigio en el ámbito de habla española, indica que "el doctorado busca analizar con recursos teóricos y metodológicos renovados las transformaciones de la realidad contemporánea, por lo que se inserta en el variado panorama de modificaciones que las ciencias sociales experimentan en las más importantes universidades del mundo" (UNAM, México, 2006). Menciona y recalca, además, el desarrollo de una investigación original desde el inicio del estudio, relacionada con la formación académica previa del aspirante.

Conscientes de la necesidad de la formación avanzada de los integrantes de las comunidades académicas y de los centros de investigación, tanto públicos como privados, se ofrecen diversas oportunidades de postgrados en Colombia y en el exterior, a través de becas manejadas por el Instituto Colombiano de Crédito Educativo y Estudios Técnicos en el Exterior (ICETEX) y COLCIENCIAS. Así, de acuerdo con Lucio (2003), el número de profesionales dedicados a la investigación en los establecimientos de educación superior, oficiales y privados, e igualmente de investigadores con "productos certificados" (artículos en revistas indexadas, libros, patentes), ha aumentado en más de un 50\%, entre el año 1998 y 2000 . Se destaca, de acuerdo al mismo autor, un incremento sustancial de 213 a 1138 investigadores con productos certificados en las Universidades Oficiales Nacionales, mientras que en las Universidades no Oficiales, de 379 investigadores con productos, en el año 1998; solamente se llegó a 448, en el año 2000. Indica el mismo autor que, en las universidades oficiales nacionales, el $34 \%$ de los docentes ostenta el título de maestría y el $8 \%$ el de doctor, mientras que en las universidades no oficiales estos porcentajes corresponden al $20 \%$ y $3 \%$, respectivamente, para el año 2000.

Una investigación original forma parte de los postgrados, tanto que la mayoría de las Universidades de la Unión Europea enfatiza en este quehacer académico. Como requisito para la obtención del título de doctor, se exige la publicación de artículos científicos en revistas seriadas indexadas.

Picardo (2002), citado por Rodríguez (2004) enfatiza que las sociedades del conocimiento deben ilustrar al público, por lo tanto, la relevancia que reviste el hacer visible los resultados es indiscutible. Mostrar a otros investigadores y al público en general los conocimien- 
tos logrados, es, por lo tanto, una obligación para los integrantes de los diversos grupos de investigación del país. Difícil es, sin embargo, llegar al público en general, ya que los medios de comunicación nacionales, como lo expresa Lisbeth Fog, no se interesan por publicar resultados de investigaciones obtenidos en el país, prefieren noticias llegadas del exterior (Mendoza, 2007). Acerca de la "apropiación social de la ciencia en la competitividad para Colombia", Solano-Franco (2005) hace un interesante análisis recalcando la ausencia de una política de divulgación, requerida para que Colombia se convierta en una sociedad del saber. El periodismo científico es una necesidad apremiante en el país.

Los profesionales, con postgrado, o no investigan o no publican, o no actualizan sus hojas de vida, lo cual, se observa con frecuencia al buscar árbitros de artículos sometidos a revistas indexadas. Hoy día, la "evaluación por pares" es una obligación y le corresponde al editor, junto con el Comité Editorial, establecer el banco de árbitros competentes (ICFES, 2003). Los pares deben cumplir requisitos para que la revista sea tenida en cuenta "para la clasificación de revistas en las categorías del índice bibliográfico nacional Publindex". Entre los requisitos, se destaca que los árbitros "deben haber publicado, en los dos años anteriores al período de observación (de la revista), al menos un artículo de los tres tipos, investigación, revisión o reflexión" (COLCIENCIAS, 2005). Al revisar en busca de árbitros las hojas de vida de los pares y de los líderes de grupos de investigación, disponibles en la página web de Colciencias, se tiene la impresión de que un título de maestría o de doctorado no faculta aparentemente para una mayor producción intelectual. Con la presente investigación, se pretendió precisamente aclarar y documentar estas hipótesis y, además, contestar las siguientes preguntas: ¿Dónde están los profesionales con postgrado en el sector privado o en el oficial de la educación superior?, cexiste diferencia en la producción, traducida en artículos científicos publicados, entre hombres y mujeres?, cंcuál de los géneros aprovecha más el postgrado?, clas universidades que se distinguen por su productividad están localizadas en el distrito capital o en la "provincia"? y, finalmente cdónde se publica más: en revistas indexadas o no indexadas?

El Observatorio Colombiano de Ciencia y Tecnología (OCyT) (2005) ya contestó algunas de estas preguntas en forma parcial, pero con un enfoque diferente, lo cual será base para el análisis de los resultados obtenidos.

\section{MATERIALES Y MÉTODOS}

Para el análisis de los indicadores bibliométricos, se escogieron las hojas de vida de los líderes de los grupos de investigación reconocidos por COLCIENCIAS, de cuatro de los once programas nacionales de Ciencia y Tecnología. Se evaluaron un total de 373 hojas de vida, 93 de líderes de los grupos del Programa Nacional de Ciencias y Tecnologías Agropecuarias, 159 de aquellos de Ciencias del Medio Ambiente y 45 y 116, respectivamente, de los Programas de Biotecnología y Estudios Científicos de la Educación (COLCIENCIAS, 2006a; 2006b; 2006c; 2006d; 2006e; 2006f). Los datos tomados fueron: la institución a que pertenece el profesional, si es pública o privada, centro de investigación, público o privado, o equivalente, el género del investigador, el máximo nivel de escolaridad y el número de publicaciones por género, tanto en revistas indexadas (COLCIENCIAS, 2005), como en no indexadas. Además, los grupos de cada programa nacional, se catalogaron en sectores. Para las ciencias y tecnologías agropecuarias, en sector agrícola y pecuaria; para ciencias del medio ambiente y hábitat, en ingenierías, biología-ecología y ecosistemas; para biotecnología en vegetal, animal y ambiental y para estudios científicos de la educación, en pedagogía, educación en ciencias y currículo. Los datos, se agruparon en tablas, para analizarlos con estadísticas descriptivas, y comparando entre sí los cuatro programas de CyT.

Para establecer la significancia estadística, se sometieron a pruebas de hipótesis, la totalidad de los datos:

Ho: $p_{1} \geq p_{2}$ donde $\mathrm{p}_{1}$ es la proporción de hombres que publican en revistas indexadas, $\mathrm{p}_{2}$ es la proporción de mujeres que publican en revistas indexadas, versus $H o: p_{1} \leq p_{2}$.La prueba efectuada fue:

$$
Z=\frac{\left(\hat{p}_{1}-\hat{p}_{2}\right)-\left(p_{1}-p_{2}\right)}{\sqrt{\frac{\hat{p}_{1}\left(1-\hat{p}_{1}\right)}{n_{1}}+\frac{\hat{p}_{2}\left(1-\hat{p}_{2}\right)}{n_{2}}}}
$$

\section{RESULTADOS Y DISCUSIÓN}

En las tablas 1, 2, 3 y 4, donde se consignaron los datos obtenidos, se observa que el programa con el menor número de grupos de investigación corresponde 
a Biotecnología (BT) (Tabla 2), seguido por el de Ciencias y Tecnologías Agropecuarias (CyTA) (Tabla 1). En comparación con los datos del OCyT (2005), se nota una disminución de grupos, de 53 a 45 en BT y de 119 a 93 en CyTA. Esto puede haber tenido la influencia negativa detectada al discutir el TLC con Estados Unidos, en el sentido de la falta de competitividad del sector. Ambos programas enfatizan sus estudios en los reinos animal y vegetal y en las perspectivas relacionadas con la biodiversidad, es decir, con lo ambiental y los sectores agrícola y vegetal. En ambos sectores sobresale la Universidad Nacional de Colombia (UN), sedes Bogotá y Medellín, seguido por las Universidades privadas, de La Salle y la Universidad de Ciencias Aplicadas y Ambientales U.D.C.A. En cuanto al programa de BT, se manifiesta una ligera superioridad de la Universidad. de Antioquia. En BT, la participación de la mujer, como líder de grupo, es del 48,8\%, mientras que en CyTA solamente alcanza un $20,4 \%$. Olaya (2003), quien analizó la participación de la mujer en la investigación, menciona que para el año 2000 , un $20 \%$ de los grupos inscritos en COLCIENCIAS eran lideradas por mujeres, y el OCyT (2005) calcula un 20,9\%; en cinco años, el panorama no ha cambiado. Con relación a las publicaciones en revistas indexadas, en BT, se destaca definitivamente la mujer. Un 68,5\% del total de las publicaciones tiene como autor principal a una mujer, mientras que en CyTA, aunque mejora un poco sobre el porcentaje de líderes de grupos, solo alcanza el 22,9\%. Si se analiza la proporción entre mujeres y hombres con educación avanzada y su producción intelectual en BT, se obtiene una proporción de 1,85:0,73. A pesar de un número considerablemente menor de mujeres con postgrados, su producción es más del doble de la de los hombres. En CyTA esta proporción corresponde a 1,06:1,10, lo cual demuestra que la mujer ha avanzado y conquistado espacios de investigación, que de acuerdo a Olaya (2003), prácticamente habían sido reservados para los hombres.

De las tablas 1 y 2 , se deduce también que una mayor actividad investigadora, se realiza en las universidades públicas. Un 18,18\% del total de instituciones involucradas del sector agropecuario corresponde a centros de investigación, dentro de los cuales se destaca CENICAFÉ. Aunque le sigue en importancia CORPOICA, se considera preocupante que esta corporación, con sedes en todo el país, dedicada de manera exclusiva a la investigación científica y tecnológica para hacer com- petitivo el sector, solamente muestra, en forma oficial, cinco grupos de investigación. Además, no se detectan artículos publicados en revistas indexadas. Igualmente llama la atención que, gremios tan importantes como los floricultores, agrupados en ASOCOFLORES, los arroceros y los palmeros, pertenecientes a FEDEARROZ y a CENIPALMA, no ostentan grupos reconocidos por COLCIENCIAS. Este aspecto se podría mejorar considerablemente al fortalecer la relación Universidad - Sector Productivo.

Un panorama diferente, se detecta en BT. Un 30,76\% de las instituciones dedicadas a la investigación, no corresponde a universidades y dentro de las 18 universidades, seis pertenecen a establecimientos privados. Se destacán la Universidad de Los Andes (U. Andes) y la Pontifica Universidad Javeriana (PUJ). La premisa de la publicación de un artículo en una revista indexada en dos años, sí se cumple en BT; mientras que en el programa agropecuario, este número ni siquiera alcanza un artículo. La proporción de publicaciones calculada entre la universidad privada y la oficial favorece en ambos programas a las privadas. En BT, la proporción es de 0,88:0,85, mientras que en CyTA corresponde a 3,00:2,36. En general existe una mayor publicación en revistas no indexadas.

Los grupos pertenecientes a Universidades localizadas en Bogotá insinúan una mayor productividad en el sector agropecuario, que aquellas situadas en las capitales de departamentos; la excepción es la U. de Antioquia. Con relación a la BT, las Universidades de "provincia", se destacan sobre las de la capital, con la excepción de la PUJ. Este último resultado, se podría deber a lo ya detectado por Lucio (2003), quien menciona que la inversión en CyT es mayor en las Universidades oficiales departamentales que en las oficiales nacionales, lo cual favorecería al programa de BT, cuyas investigaciones requieren inversiones elevadas, tanto en infraestructura como en equipos.

En la tabla 3, se encuentran los resultados del programa de Ciencias del Medio Ambiente y Hábitat (CMAH). Nuevamente, se nota una disminución en el número de grupos, de 187 reconocidos en el año 2005 (OCyT, 2005) quedaron solo 159, en el 2006. Los grupos, se dividieron en Ingenierías, Biología y Ecología y Ecosistemas; aquellos dedicados a Ingenierías ocupan el mayor 
Tabla 1. Programa Nacional de Ciencias y Tecnologías Agropecuarias. Producción intelectual con relación al género y el último título académico obtenido*.

\begin{tabular}{|c|c|c|c|c|c|c|c|c|c|c|c|}
\hline \multirow{3}{*}{$\begin{array}{l}\text { INSTITUCIÓN } \\
\text { U.N. BOGOTÁA }{ }^{* *}\end{array}$} & \multirow{2}{*}{\multicolumn{2}{|c|}{$\begin{array}{l}\text { GRUPOS/SECTOR } \\
\text { Agríc. Pec. }\end{array}$}} & \multicolumn{2}{|c|}{ GÉNERO } & \multicolumn{4}{|c|}{ POSTGRADO** } & \multicolumn{3}{|c|}{$\begin{array}{l}\text { PUBL. EN REVISTAS } \\
2004-2005^{\star * *}\end{array}$} \\
\hline & & & & Q & 0 & ధ & 3 & Q & & x. & dex. \\
\hline & 11 & 9 & 17 & 3 & 4 & 3 & 12 & & 18 & 1 & 2 \\
\hline U.N. MEDELLIN & 4 & 1 & 5 & & & & 5 & & 4 & & \\
\hline U.N. PALMIRA & 2 & 1 & 3 & & 1 & & 2 & & 5 & & \\
\hline U.N. MANIZALES & 1 & & 1 & & & & 1 & & & & \\
\hline U. DE NARIÑO & 1 & 1 & 2 & & 1 & & 1 & & 2 & & \\
\hline U. DEL CAUCA & 3 & 1 & 2 & 2 & 1 & 2 & 1 & & & & \\
\hline U. DEL QUINDIOO & 1 & & 1 & & & & 1 & & 2 & & \\
\hline U. DEL VALLE & 1 & & 1 & & & & 1 & & & & \\
\hline $\begin{array}{l}\text { U. DE FRANCISCO DE } \\
\text { P. SANTANDER }\end{array}$ & 1 & & & 1 & & 1 & & & & & \\
\hline U.P.T.C TUNJA** & 3 & 1 & 4 & & 2 & & 1 & & 6 & & \\
\hline U. DE ANTIOQUIA & 1 & 3 & 1 & 3 & & 1 & 1 & 2 & 3 & 6 & 2 \\
\hline U. DE CALDAS & 1 & 3 & 4 & & 1 & & 3 & & & & 1 \\
\hline U. DE LA SALLE & 1 & 5 & 3 & 3 & 3 & 2 & 1 & & 3 & 3 & 4 \\
\hline U. DE CORDOBA & 1 & 1 & 2 & & 1 & & 1 & & 5 & & 1 \\
\hline U.D.C.A $A^{\star * \star \star}$ & 2 & 3 & 2 & 3 & 1 & 1 & & 1 & 5 & 2 & 1 \\
\hline U. T. DEL CHOCO ${ }^{* *}$ & 2 & & 1 & 1 & & & & 1 & & 1 & 4 \\
\hline UIS & 1 & & & 1 & & & 1 & & & & 2 \\
\hline CORP. UNIV. SANTA & 1 & & 1 & & & & & & & & 4 \\
\hline $\begin{array}{l}\text { ROSA DE CABAL } \\
\text { U. J. TADEO LOZANO }\end{array}$ & 2 & & 2 & & & & 2 & & 5 & & \\
\hline $\begin{array}{l}\text { U. POPULAR DEL } \\
\text { CESAR }^{* *}\end{array}$ & 1 & & 1 & & & & & & & & 1 \\
\hline $\begin{array}{l}\text { CORP. UNIV. DE } \\
\text { SANTANDER }\end{array}$ & 1 & & 1 & & & & & & & & \\
\hline U. DE CARTAGENA & 1 & & 1 & & 1 & & & & & & 1 \\
\hline UNISUR & 1 & & 1 & & & & 1 & & & & \\
\hline U. DEL TOLIMA & 1 & & 1 & & & & 1 & & & & \\
\hline U. SANTO TOMÁS & 1 & & 1 & & 1 & & & & & & \\
\hline U.DEL MAGDALENA & 2 & & 2 & & 1 & & 1 & & & & 1 \\
\hline $\begin{array}{l}\text { CENTRO INV. DEL } \\
\text { BANANO AUGURA }\end{array}$ & 2 & & 2 & & 2 & & & & & & 1 \\
\hline CENICAFÉ & 5 & 1 & 6 & & 1 & & 4 & & 16 & & 2 \\
\hline CORPOICA & 1 & 4 & 4 & 1 & & 1 & 4 & & & & 1 \\
\hline CONIF ${ }^{\star \star}$ & 1 & & 1 & & & & & & & & \\
\hline CENICAÑA & 1 & & 1 & & & & 1 & & & & \\
\hline SINCHI & 1 & & & 1 & & & & 1 & & 4 & \\
\hline TOTAL & 59 & 34 & 74 & 19 & 21 & 11 & 46 & 5 & 74 & 17 & 28 \\
\hline
\end{tabular}

* Resumen de datos tomados de la hoja de vida de los líderes de los 93 grupos de investigación reconocidos por COLCIENCIAS (enero de 2006).

** Diez líderes sin postgrado.

*** Se incluyeron artículos aceptados para publicación. 
Tabla 2. Programa Nacional Biotecnología. Producción intelectual con relación al género y el último título académico obtenido*.

\begin{tabular}{|c|c|c|c|c|c|c|c|c|c|c|c|c|}
\hline INSTITUCIÓN & $\begin{array}{l}\text { GRU } \\
\text { Veg. }\end{array}$ & $\begin{array}{l}\text { /SEC } \\
\text { nim. }\end{array}$ & & $\begin{array}{l}\text { GÉI } \\
\sigma^{\prime \prime}\end{array}$ & Q & & & $\begin{array}{l}A D \\
D \\
0\end{array}$ & q & & $\begin{array}{l}\text { L. E } \\
004 \\
x . \\
\text { O }\end{array}$ & $\begin{array}{l}\text { REVISTAS } \\
2005^{\star * *} \\
\text { No index. }\end{array}$ \\
\hline U.N. BOGOTÁ & 2 & 1 & 1 & 2 & 2 & & 1 & 2 & 1 & 1 & 1 & 1 \\
\hline U.N. MEDELLIN & 1 & 2 & 2 & 2 & 3 & 1 & 2 & 1 & 1 & 1 & 5 & \\
\hline U. DEL CAUCA & 1 & & & 1 & & 1 & & & & & & 3 \\
\hline U. DEL VALLE & & 1 & & 1 & & & & 1 & & 1 & & 1 \\
\hline U.P.T.C TUNJA & & & 1 & & 1 & & & & 1 & & 1 & \\
\hline U. DE ANTIOQUIA & 2 & 3 & 1 & 2 & 4 & 1 & 1 & 1 & 3 & 2 & 2 & 1 \\
\hline U. DE LA SALLE & 1 & 1 & 1 & 2 & 1 & 1 & & 1 & 1 & 1 & & 2 \\
\hline U.T. DEL CHOCÓ & 1 & & & 1 & & & & 1 & & & & \\
\hline UIS & & 1 & & 1 & & & & 1 & & 1 & & \\
\hline U.DEL MAGDALENA & 1 & 1 & & 1 & 1 & 1 & & & 1 & & 5 & \\
\hline U. DE LOS ANDES & & & 2 & & 2 & & 1 & & 1 & & & \\
\hline PONT. U. JAVERIANA & 2 & & 1 & 2 & 1 & 1 & & 1 & 1 & 5 & & 1 \\
\hline U. PONT. BOLIVARIANA & & & 1 & & 1 & & & & 1 & & & 4 \\
\hline U. CATOL. ORIENTE & 1 & & & 1 & & & & 1 & & & & \\
\hline U. TECN. PEREIRA & 1 & & & & 1 & & & & 1 & & 1 & \\
\hline U. MIL. N. GRANADA & 1 & & & & 1 & & & & 1 & & & \\
\hline U. CAT. MANIZALES & 1 & & & 1 & & & & 1 & & 1 & & \\
\hline U. M. BELTRÁN & & 1 & & 1 & & 1 & & & & & & \\
\hline CIB & 1 & 1 & & 2 & & & & 2 & & 4 & & \\
\hline CORPOICA & & & 1 & & 1 & & & & 1 & & 19 & \\
\hline CORP. BIOTEC & 1 & & & 1 & & & & 1 & & & & \\
\hline FUND. TROPILOGÍA** & & 1 & & 1 & & & & & & & & \\
\hline CORPOGEN ** & & 1 & & & 1 & & & & & & 3 & \\
\hline $\begin{array}{l}\text { CENTRO INTERNAL } \\
\text { FÍSICA }\end{array}$ & 1 & & & & 1 & & 1 & & & & & \\
\hline ICA & 1 & & & 1 & & & & 1 & & & & 3 \\
\hline POLICÍA NAL. JUDICIAL & & 1 & & & 1 & 1 & & & & & & \\
\hline TOTAL & 19 & 15 & 11 & 23 & 22 & 8 & 6 & 15 & 14 & 17 & 37 & 16 \\
\hline
\end{tabular}

* Resumen de datos tomados de la hoja de vida de los líderes de los 45 grupos de investigación reconocidos por COLCIENCIAS (enero de 2006).

** Diez líderes sin postgrado.

*** Se incluyeron artículos aceptados para publicación.

porcentaje, 39,62\%, lo cual se refleja también en la producción intelectual por género. Solamente, un $29,7 \%$ de los artículos publicados en revistas indexadas tiene como autores a mujeres. Sin embargo, la proporción calculada de artículos durante dos años entre mujeres y hombres con título de postgrado, muestra una ligera ventaja del género femenino, 0,83 sobre 0,80. En este programa que representa a los grupos dedicados a los aspectos ecológicos, biológicos y a los ecosistemas, se suponía una mayor participación de la mujer, lo cual todavía no se vislumbra, solo un $28,30 \%$ de los grupos es liderado por el género femenino.
La proporción de la producción entre los investigadores de las Universidades públicas, privadas y las instituciones o centros de investigación, corresponde a 2,75:2,2:0,53. Se destaca la Universidad pública, tanto de la capital, como de la provincia, sobresaliendo nuevamente la U. de Antioquia, seguida por la U.N. sede Bogotá y sede Medellin, la U. del Valle y la U. Industrial de Santander (UIS). En cuanto a las universidades privadas, se destaca la U. Andes, seguida por la PUJ. Finalmente, sobresale en forma negativa, que los líderes de los 159 grupos solamente son coautores de un total de 121 artículos, no alcanzando la meta de un artículo por dos años. Sin 
Tabla 3. Programa Nacional de Ciencias del Medio Ambiente y Hábitat. Producción intelectual con relación al género y el último título académico obtenido*.

\begin{tabular}{|c|c|c|c|c|c|c|c|c|c|c|c|c|}
\hline \multirow[t]{2}{*}{ INSTITUCIÓN } & \multicolumn{3}{|c|}{ GRUPOS/SECTOR } & \multicolumn{2}{|c|}{ GÉNERO } & \multicolumn{4}{|c|}{$\begin{array}{l}\text { POSGRADO** } \\
\text { M.Sc. Ph.D }\end{array}$} & \multicolumn{3}{|c|}{$\begin{array}{c}\text { PUBL. EN REVISTAS } \\
2004-2005^{* * *}\end{array}$} \\
\hline & Ing. & $\begin{array}{l}\text { Biol. } \\
\text { Ecosist. }\end{array}$ & Ecol. & $\sigma$ & @ & $\sigma^{\prime \prime}$ & Q & $\sigma^{\prime \prime}$ & q & & 우 & No index. \\
\hline U.N. BOGOTÁ & 5 & 7 & 3 & 13 & 2 & 6 & 2 & 7 & & 14 & & 1 \\
\hline U.N. MEDELLIIN & 2 & 1 & 5 & 4 & 4 & & 2 & 4 & 2 & 7 & 2 & 1 \\
\hline U.N. PALMIRA & & & 1 & 1 & & & & 1 & & & & \\
\hline U.N. MANIZALES & 1 & & 3 & 3 & 1 & 1 & & 2 & 1 & 2 & & \\
\hline U.N. LETICIA & & 1 & & 1 & & 1 & & & & 5 & & \\
\hline EAFIT** & 3 & & & 3 & & 2 & & & & 5 & & 4 \\
\hline U. DEL CAUCA & & 5 & & 3 & 2 & 2 & 1 & 1 & 1 & & & 2 \\
\hline U. DEL QUINDIOO & & 1 & & 1 & & 1 & & & & & & \\
\hline U. DEL VALLE & 7 & 1 & 3 & 7 & 4 & 2 & & 5 & 4 & 1 & 7 & 2 \\
\hline $\begin{array}{l}\text { U. DE FRANCISCO DE P. } \\
\text { SANTANDER }\end{array}$ & 1 & & & & 1 & & & & 1 & & & \\
\hline U.P.T.C TUNJA & 1 & 2 & 1 & 3 & 1 & 1 & 1 & 2 & & 1 & & 1 \\
\hline U. DE ANTIOQUIA & 4 & 3 & 1 & 3 & 5 & 1 & & 2 & 5 & 3 & 19 & 1 \\
\hline U. DE CALDAS & & & 1 & 1 & & & & 1 & & & & 2 \\
\hline U.D.C.A ** & & 1 & & & 1 & & & & & & 2 & \\
\hline U. TÉC. DEL CHOCÓ ** & 1 & 5 & & 4 & 2 & 3 & 2 & & & 2 & & 2 \\
\hline UIS & 2 & & & 1 & 1 & & & 1 & 1 & 1 & 5 & \\
\hline $\begin{array}{l}\text { Fund. U. J. TADEO } \\
\text { LOZANO }\end{array}$ & & 2 & & & 2 & & 2 & & & & & \\
\hline U. DEL BOSQUE & 1 & & & 1 & & & & 1 & & & & \\
\hline U. DE CARTAGENA & 1 & & & 1 & & & & 1 & & 4 & & \\
\hline UNISUR & & 1 & & 1 & & & & 1 & & & & 1 \\
\hline U. DEL TOLIMA & & & 1 & 1 & & & & 1 & & & & \\
\hline U. SANTO TOMÁS & 1 & & 1 & 2 & & 2 & & & & & & 1 \\
\hline U. DEL MAGDALENA & 2 & 1 & 2 & 4 & 1 & 2 & 1 & 2 & & 1 & & 1 \\
\hline $\begin{array}{l}\text { ESC. COL. ING. JULIO. } \\
\text { GARAVITO }\end{array}$ & 5 & & & 5 & & 4 & & 1 & & 5 & & 2 \\
\hline U. DE LOS ANDES & 3 & & 5 & 8 & & 4 & & 4 & & 10 & & 1 \\
\hline PONT. U. JAVERIANA & 4 & 1 & 4 & 4 & 5 & 1 & 1 & 3 & 4 & 4 & 3 & 1 \\
\hline $\begin{array}{l}\text { U. AUTÓNOMA DEL } \\
\text { OCCIDENTE }\end{array}$ & & 1 & & & 1 & & & & 1 & & & 3 \\
\hline $\begin{array}{l}\text { FUNDACIÓN UNIV. DEL } \\
\text { NORTE ** }\end{array}$ & 3 & & & 3 & & 1 & & 1 & & 3 & & 1 \\
\hline U. DIST. F.J. CALDAS & 1 & 1 & 1 & 3 & & 3 & & & & 2 & & \\
\hline U. TECN. DE PEREIRA & 2 & & 2 & 4 & & 1 & & 3 & & 4 & & \\
\hline U. ANTONIO NARIÑO & 1 & & 1 & 1 & 1 & & 1 & 1 & & & & 1 \\
\hline U. DEL ATLÁNTICO & 1 & & 1 & 1 & 1 & 1 & & & 1 & & & \\
\hline U. PILOTO DE COL. ** & & & 2 & 2 & & 1 & & & & & & \\
\hline U. MIL.N. GRANADA & 1 & & 1 & & 2 & & 1 & & 1 & & 1 & \\
\hline U DE BOYACÁ ** ** & 2 & & 1 & 2 & 1 & 1 & & & & 1 & & 1 \\
\hline U. PONTIF. BOLIV. & 2 & & & 1 & 1 & 1 & 1 & & & & & \\
\hline ESC. ING. ANTIOQU. & 1 & & & 1 & & 1 & & & & 1 & & 1 \\
\hline U. DE MEDELLÍN & 1 & & & & 1 & & & & 1 & & 2 & \\
\hline U. SANTIAGO CALI & & 1 & & 1 & & 1 & & & & & & 2 \\
\hline U. DE SUCRE & & 1 & & 1 & & 1 & & & & & & \\
\hline U. DE MANIZALES & 1 & & & 1 & & 1 & & & & 1 & & 3 \\
\hline PONT. U. JAV. CALI & & & 1 & 1 & & & & 1 & & 1 & & \\
\hline U. EXTERN. DE COL. & & & 1 & 1 & & & & 1 & & 2 & & \\
\hline $\mathrm{CIPAV}^{* *}$ & & & 1 & 1 & & & & & & & & \\
\hline
\end{tabular}


Tabla 3. (Continuación)

\begin{tabular}{|c|c|c|c|c|c|c|c|c|c|c|c|c|}
\hline \multirow[t]{2}{*}{ INSTITUCIÓN } & \multicolumn{3}{|c|}{ GRUPOS/SECTOR } & \multicolumn{2}{|c|}{ GÉNERO } & \multicolumn{4}{|c|}{$\begin{array}{l}\text { POSGRADO** } \\
\text { M.Sc. Ph.D }\end{array}$} & \multicolumn{3}{|c|}{$\begin{array}{c}\text { PUBL. EN REVISTAS } \\
2004-2005^{\star * *}\end{array}$} \\
\hline & Ing & $\begin{array}{l}\text { Biol. } \\
\text { Ecosist }\end{array}$ & Ecol. & $\sigma^{\prime \prime}$ & Q & $\sigma$ & Q & $\sigma$ & Q & & ex. & No index. \\
\hline INGEOMINAS & & & 3 & 2 & 1 & 1 & & 1 & 1 & & & \\
\hline$J \&$ G ASOCIADOS & 1 & & & 1 & & & & 1 & & 2 & & \\
\hline \begin{tabular}{|l} 
INST. NAL. SALUD \\
\end{tabular} & & & 1 & & 1 & & & & 1 & & & \\
\hline $\begin{array}{l}\text { INST. TECN. METROP. } \\
\text { MEDELLIIN }\end{array}$ & 1 & & & 1 & & & & 1 & & & & 3 \\
\hline SINCHI ** & & 1 & 3 & 4 & & 2 & & 1 & & 2 & & \\
\hline FUND. NATURA COL. & & & 1 & & 1 & & 1 & & & & & \\
\hline CORPOICA & & & 1 & 1 & & & & 1 & & & & \\
\hline $\begin{array}{l}\text { INST. INVEST. PRESERV. } \\
\text { PATRIM. }\end{array}$ & & 1 & & 1 & & & & 1 & & & & \\
\hline $\begin{array}{l}\text { CORP.C.I. CARBONO \& } \\
\text { BOSQUES MED.** }\end{array}$ & & 1 & & 1 & & & & & & & & \\
\hline \begin{tabular}{|l|} 
INVEMAR \\
\end{tabular} & & & 1 & 1 & & & & 1 & & & & \\
\hline CENICAFÉ & & 1 & & 1 & & & & 1 & & 1 & & 2 \\
\hline FUND.ARAGUATOS** & & 1 & & 1 & & & & & & 1 & & \\
\hline U. DE PAMPLONA & 1 & & & & 1 & & 1 & & & & & \\
\hline $\begin{array}{l}\text { INST. ALEXANDER VON } \\
\text { HUMBOLDT }\end{array}$ & & 1 & & & 1 & & & & 1 & & 2 & 1 \\
\hline $\begin{array}{l}\text { CORP. OBSERVAT. DEL } \\
\text { CARIBE }\end{array}$ & & & 1 & 1 & & 1 & & & & & & \\
\hline TOTAL & 63 & 42 & 54 & 114 & 45 & 49 & 17 & 56 & 26 & 85 & 36 & 41 \\
\hline
\end{tabular}

* Resumen de datos tomados de la hoja de vida de los líderes de los 159 grupos de investigación reconocidos por COLCIENCIAS (enero de 2006).

** Once líderes sin postgrado.

*** Se incluyeron artículos aceptados para publicación.

embargo, al incluir los artículos publicados en revistas no indexadas, si se lograra por lo menos un artículo en dos años, lo cual sigue siendo una pobre producción.

Al comparar estos datos con aquellos del análisis bibliométrico durante cinco años sobre la actividad investigativa en Biodiversidad (Biosintesis, 2001), se observa una aparente disminución de la producción de los investigadores en este campo. El total de artículos, en el periodo comprendido entre 1995 y 1999, fue de 1084 en revistas nacionales e internacionales, indexadas o no, mientras que en dos años, incluyendo los tres sectores en los cuales se dividió el Programa Nacional de $\mathrm{CMAH}$, el cual incluye la Biodiversidad, solamente se detectaron 162 artículos.

El último programa, el de Estudios Científicos de la Educación (ECE) (Tabla 4), muestra un panorama desolador. Los líderes de los 116 grupos de investigación, 38 menos que en el año 2005 (OCyT, 2005), quienes se ocupan de los sectores de la pedagogía, de la educación en ciencias y de los aspectos curriculares, nueve sin postgrado, 51 con título de maestría y 56 con doctorado, durante los últimos dos años solamente publicaron 40 escritos en revistas indexadas. Sumando lo publicado en revistas indexadas y no, la producción corresponde a medio artículo por año. El Programa de ECE es el único de CyT en el cual el número de mujeres con doctorado sobrepasa el de los hombres, lo cual ya fue detectado por Tovar (2002). Sin embargo, solamente un 35\% de los resultados visibles de las investigaciones es fruto del género femenino.

En este programa sobresalen los productos de las Universidades públicas, encabezado por la U. Pedagógica Nacional, seguido por la U. Distrital Francisco José de Caldas y las Universidades del Atlántico y de Antioquia. Las Universidades privadas, aunque muestran una participación por grupos del $24,13 \%$, solamente ostentan el $12,5 \%$ del total de publicaciones, destacándose 
Tabla 4. Programa Nacional de Estudios Científicos de la Educación. Producción intelectual con relación al género y el último título académico obtenido*.

\begin{tabular}{|c|c|c|c|c|c|c|c|c|c|c|c|c|}
\hline \multirow[t]{2}{*}{$\begin{array}{l}\text { INSTITUCIÓN } \\
\text { U.N. BOGOTÁA }{ }^{* *}\end{array}$} & \multicolumn{3}{|c|}{$\begin{array}{l}\text { GRUPOS/SECTOR } \\
\text { Pedag. Educac. Curr. } \\
\text { en Cienc. }\end{array}$} & \multicolumn{2}{|c|}{ 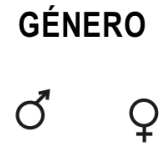 } & \multicolumn{4}{|c|}{ 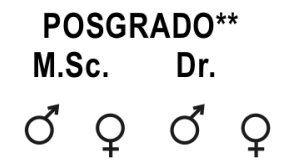 } & \multicolumn{3}{|c|}{$\begin{array}{l}\text { PUBL. EN REVISTAS } \\
2004-2005^{* * *} \\
\text { Index. No index. } \\
\sigma \quad \%\end{array}$} \\
\hline & 1 & 2 & & 3 & & 1 & & 1 & & 1 & & \\
\hline $\begin{array}{l}\text { U.N. ABIERTA Y A } \\
\text { DISTANCIA }\end{array}$ & 1 & & & 1 & & & & 1 & & 1 & & \\
\hline U.N. MANIZALES ** & & 1 & 1 & 2 & & 1 & & & & & & \\
\hline U. DE NARIÑO & & & 1 & & 1 & & 1 & & & & & \\
\hline U. DEL CAUCA ** & 2 & 1 & 1 & 1 & 3 & & & 1 & 2 & & 2 & 4 \\
\hline U. DEL VALLE & 2 & 5 & & 4 & 3 & 3 & & 1 & 3 & & & 1 \\
\hline $\begin{array}{l}\text { U. FRANCISCO DE P. } \\
\text { SANTANDER ** }\end{array}$ & 1 & & & 1 & & & & & & & & \\
\hline U.P.T.C TUNJA & 1 & & & 1 & & & & 1 & & 1 & & 1 \\
\hline U. DE ANTIOQUIA & 8 & 3 & 2 & 6 & 7 & & 3 & 4 & 6 & 4 & & 4 \\
\hline U. DE CALDAS & & 2 & 1 & 2 & 1 & & & 2 & 1 & 1 & & 1 \\
\hline U. T. DEL CHOCÓ & 1 & & & & 1 & & & & 1 & & & 1 \\
\hline UIS & 1 & 1 & & & 2 & & & & 2 & & & \\
\hline U. DE CARTAGENA & 1 & & & & 1 & & & & 1 & & 1 & 2 \\
\hline UNISUR & & & 2 & 2 & & 1 & & 1 & & & & 1 \\
\hline U. SANTO TOMÁS & 1 & 1 & & 2 & & & & 2 & & & & 4 \\
\hline U. DEL MAGDALENA ** & 1 & & 2 & 1 & 2 & 1 & 1 & & & 2 & & 4 \\
\hline $\begin{array}{l}\text { ESC. COL. ING. JULIO } \\
\text { GARAVITO }\end{array}$ & & 1 & & 1 & & 1 & & & & & & \\
\hline U. DE LOS ANDES & 1 & 2 & & 2 & 1 & 1 & & 1 & 1 & 2 & & \\
\hline PONT. U. JAVERIANA ** & 1 & 2 & & 3 & & 1 & & & & & & \\
\hline FUND. U. DEL NORTE & 1 & 1 & & 1 & 1 & & & 1 & 1 & & & 1 \\
\hline U.DIST. F. J. CALDAS ** & 6 & 5 & & 2 & 9 & & 4 & 1 & 5 & 2 & 5 & 4 \\
\hline U.T. DE PEREIRA ** & 2 & & 1 & 1 & 2 & & & & 2 & 1 & 1 & \\
\hline U. ANTONIO NARIÑO & 1 & & & & 1 & & & & 1 & & & \\
\hline U. DEL ATLÁNTICO & 6 & & 1 & 4 & 3 & 2 & 1 & 2 & 2 & 4 & & 3 \\
\hline U. PEDAG. NAL. & 4 & 5 & 2 & 4 & 7 & 2 & 5 & 2 & 2 & 3 & 5 & 3 \\
\hline U. MIL. N. GRANADA & & 1 & & & 1 & & & & 1 & & & 2 \\
\hline U DE LA SALLE & 1 & & & 1 & & 1 & & & & 1 & & 3 \\
\hline U. PONTIV. BOLIV. & 1 & & & & 1 & & & & 1 & & & \\
\hline U. CAT. ORIENTE & 1 & & & 1 & & & & 1 & & & & \\
\hline $\begin{array}{l}\text { U. AUTÓNOMA } \\
\text { MANIZALES }\end{array}$ & & & 1 & 1 & & & & 1 & & 1 & & \\
\hline U. CENTRAL & 1 & & & 1 & & 1 & & & & & & \\
\hline $\begin{array}{l}\text { U. COOP. DE COLOMBIA } \\
\text { (Bogotá) }\end{array}$ & 1 & & & & 1 & & & & 1 & & & \\
\hline U. DE PAMPLONA & & 1 & & & 1 & & 1 & & & & & 1 \\
\hline U. DE LA SABANA & 1 & & & 1 & & & & 1 & & 1 & & \\
\hline U. DE S. BUENAVENT. & 1 & & & & 1 & & 1 & & & & & \\
\hline U. LIBRE COL. (Cali) & 1 & & & 1 & & 1 & & & & & & 1 \\
\hline $\begin{array}{l}\text { ESC. PEDAG. } \\
\text { EXPERIMENTAL }\end{array}$ & 1 & 2 & & 3 & & 3 & & & & & & \\
\hline COOP. UNIV. SINU & 1 & & & & 1 & & 1 & & & & & \\
\hline U. EXTERN. DE COL. & & & 1 & & 1 & & 1 & & & & & \\
\hline
\end{tabular}


Tabla 4. (Continuacioón)

\begin{tabular}{|c|c|c|c|c|c|c|c|c|c|c|c|c|}
\hline \multirow[t]{2}{*}{ INSTITUCIÓN } & \multirow{2}{*}{\multicolumn{3}{|c|}{$\begin{array}{l}\text { GRUPOS/SECTOR } \\
\text { Pedag. Educac. Curr. } \\
\text { en Cienc. }\end{array}$}} & \multicolumn{2}{|c|}{ GÉNERO } & \multicolumn{4}{|c|}{$\begin{array}{l}\text { POSGRADO** } \\
\text { M.Sc. Dr. }\end{array}$} & \multicolumn{3}{|c|}{$\begin{array}{c}\text { PUBL. EN REVISTAS } \\
2004-2005^{* * *}\end{array}$} \\
\hline & & & & & Q & & ధ & 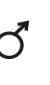 & Q & & $\begin{array}{l}\text { x. } \\
\text { o }\end{array}$ & dex. \\
\hline $\begin{array}{l}\text { CORP. UNIVERSITARIA } \\
\text { MINUTO DE DIOS }\end{array}$ & 1 & & & 1 & & 1 & & & & & & \\
\hline $\begin{array}{l}\text { U. COOP. DE COLOMBIA } \\
\text { (Barancabermeja) }\end{array}$ & 1 & & & & 1 & & 1 & & & & & 1 \\
\hline $\begin{array}{l}\text { CORP. SOC. COL. DE } \\
\text { PEDAGOGÍA }\end{array}$ & 1 & & & 1 & & 1 & & & & & & \\
\hline $\begin{array}{l}\text { CORP. EDUC.MAYOR DEL } \\
\text { DESARROLLO S. BOLIVAR }\end{array}$ & 1 & 2 & & 2 & 1 & 1 & & 1 & 1 & & & 18 \\
\hline $\begin{array}{l}\text { FUND.REVISTA.EDUC. } \\
\text { CIENCIAS }\end{array}$ & & 1 & & 1 & & & & 1 & & 1 & & \\
\hline $\begin{array}{l}\text { FUND. CAMINOS DE } \\
\text { ENTIDAD }\end{array}$ & 1 & & & 1 & & 1 & & & & & & \\
\hline INST. NAL. SALUD & 1 & & & 1 & & 1 & & & & & & 1 \\
\hline ASCUN & 1 & & & & 1 & & 1 & & & & & 1 \\
\hline CENTRO DE INVEST. Y & & 1 & & & 1 & & & & 1 & & & 1 \\
\hline DES. ACADÉMICO & & & & & & & & & & & & \\
\hline TOTAL & 60 & 40 & 16 & 60 & 56 & 25 & 21 & 26 & 35 & 26 & 14 & 63 \\
\hline
\end{tabular}

* Resumen de datos tomados de la hoja de vida de los líderes de los 159 grupos de investigación reconocidos por COLCIENCIAS (enero de 2006).

** Once líderes sin postgrado.

*** Se incluyeron artículos aceptados para publicación.

nuevamente la U. Andes y mostrando su participación la U. de La Sabana. No se detectó diferencia significativa entre las Universidades con sede en el Distrito Capital y en la Provincia. La ausencia de la visibilidad de los resultados, se puede considerar alarmante en este sector, que abarca no solamente la educación superior sino todos los niveles educacionales que conducen a ella. Murcia (2004) atribuye en parte la aparente carencia de investigación educativa a la deficiencia educacional de metodologías de investigación en el pregrado y la falta de incentivos. Igualmente, menciona la autora, la falta de personal especializado en investigación para el año 2000, lo cual, basado en el elevado número de grupos actualmente reconocidos por COLCIENCIAS, ya pierde vigencia. Rodríguez (2004), analizando los "problemas que afectan el desarrollo del conocimiento", sugiere una reorientación de la investigación en educación.

Rosselli-Cock (2005), al buscar las causas de la reducida publicación científica colombiana, menciona el poco énfasis "en la redacción" en las instituciones de educación superior, en comparación con las universidades anglosajonas. Analizando el plan de estudios de diversos programas de maestría y doctorado en el país, efectivamente éstos carecen de asignaturas que "enseñan a escribir". ¿Será que el miedo al ver un artículo rechazado, impide una mayor producción intelectual de los investigadores colombianos?

Las pruebas de hipótesis comprobaron lo indicado anteriormente. Para los líderes de los grupos agropecuarios, se rechaza la $\mathrm{H}_{\mathrm{o}}$, a un nivel de significancia del $10 \%$, es decir, si existe diferencia en publicaciones en revistas indexadas y los hombres no publican más que las mujeres. Para el Programa CMAH, la prueba proporciona -3,968. La hipótesis nula es también rechazada y se acepta la alternativa: el género masculino no publica más que el femenino. Para el tercer programa, la BT, el Z obtenido -0,64 hace que aceptamos la hipótesis nula: es decir, proporcionalmente los hombres muestran una mayor visibilidad de sus investigaciones. Los líderes de los grupos del Programa ECE, con $Z=2,05$, muestran nuevamente la superioridad proporcional por parte de las mujeres.

Para el integrado de los anteriores programas, la prueba proporciona 2,08, lo cual implica, en forma global, el rechazo de la hipótesis nula, es decir, los hombres no 
publican más que mujeres. Este hallazgo final de una mayor productividad de las mujeres, líderes de grupos se puede deber, en general, a la mayor dedicación de este género. Probablemente, avanzar en el mundo competitivo de CyT y escalar posiciones, ha sido difícil y, una vez alcanzado, ellas quieren demostrar que merecen estas distinciones.

Al organizar las instituciones en públicas y privadas y proponer la misma hipótesis, se obtiene que, para el caso del sector público, la $Z$ nos da: 6,374 y para el sector privado: $-1,58$. Del $100 \%$ de los grupos analizados, en el sector privado, el $11,11 \%$ es liderado por hombres y $20,29 \%$ por mujeres, y en el sector público el 36,47\% y el 32,13\%, respectivamente. La participación de la mujer en grupos y centros de investigación, en los dos sectores, representó de acuerdo a Olaya (2003) un 40\%; lo cual, para los programas analizados, muestra un avance en la participación de la mujer en CyT.
Referente al título académico, maestría o doctorado, el $36,71 \%$ de los líderes pertenece al sector privado y el $63,29 \%$, al sector público; el $12,66 \%$ son hombres del sector privado y el $28,48 \%$ del público, mientras que el $24,05 \%$ de las mujeres son del sector privado y el $34,81 \%$ del público. Respecto al doctorado éstos corresponden al $23,42 \%$ del sector privado y al 75,58\% del público, el $9 \%$ de los hombres son del privado y el $45,5 \%$ del público y las mujeres son $14,41 \%$ del sector privado y el 31,08\% del sector público. Al hacer referencia a las publicaciones en revistas indexadas, el 24,6\% es producido por investigadores de entidades privadas y el $75,4 \%$ por profesionales de entidades públicas. El género femenino solamente representa alrededor del $50 \%$ en todas las categorías mostradas en la figura 1 , lo cual indica, sin embargo, un aumento del porcentaje de mujeres con postgrados, en relación con lo detectado por Tovar (2002), el 42,6\%.

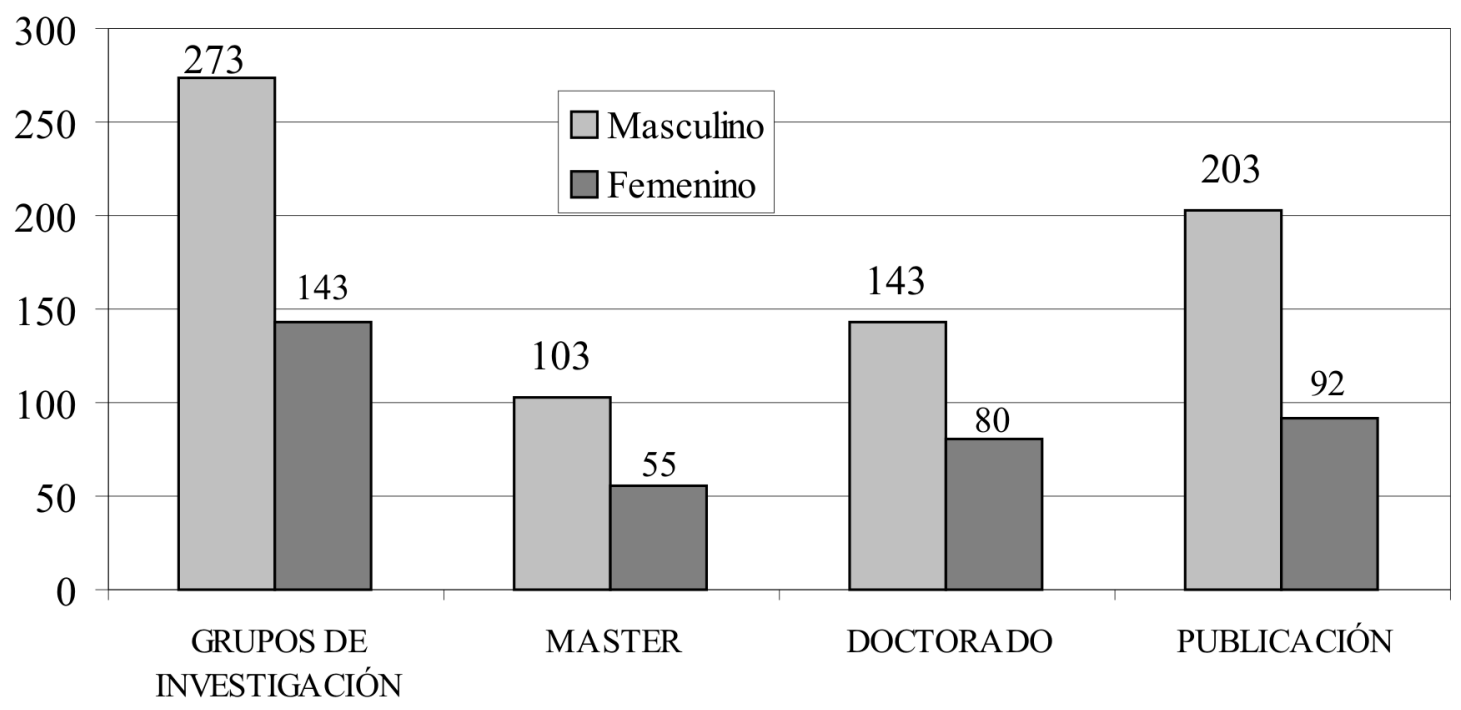

Figura 1: Análisis comparativo del comportamiento del género del líder del grupo de investigación, su grado académico y su producción intelectual.

Para todos los grupos de investigación inscritos para enero del año 2005, OCyT calculó 2,19 líderes hombres por cada líder mujer. En este estudio, enero 2006, para los cuatro programas la relación correspondió a
1,96:1, lo cual se podría interpretar como un avance en la participación del género femenino como directrices de grupos. Al realizar la misma comparación para postgrados, el OCyT muestra 2,77 hombres doctores por 
cada mujer con este título, mientras que para los cuatro programas analizados esta relación es más favorable al género femenino, mostrando una proporción de 1,78 doctores hombres por cada mujer con este título.

Con estos resultados preliminares del avance de la mujer en los diversos campos de la ciencia, se vislumbra un futuro más prometedor para el género, lo cual concuerda, en general, con lo expuesto por González \& Pérez (2002), quienes afirman que ya existe una integración, por lo menos parcial, de las mujeres a campos previamente vedados. Además, resaltan que este aspecto "implica un claro compromiso con la transformación de la educación, la práctica y la gestión de la ciencia y tecnología".

Los resultados indican que, en general y a pesar de la supremacía del sector público en cuanto a la existencia de grupos de investigación reconocidos, este sector no sobresale proporcionalmente en productividad intelectual. La inversión en investigación, traducido en infraestructura y postgrados, se debería reflejar en una mayor visibilidad de resultados. Es probable que en la universidad pública muchos docentes buscan un postgrado, ante todo, para fines de avanzar en el escalafón. Además, se puede concluir que:

- Los postgrados no incentivaron a los tenedores de los títulos, maestría o doctorado para una mayor producción y divulgación de conocimientos.

- Un título aparentemente tiene como meta principal el avance en el escalafón, establecido por el Decreto 1279 del 2002 para los docentes de las Universidades Estatales y no la búsqueda de nuevos conocimientos a través de la investigación. Este "avance" es igualmente válido para las Universidades privadas y centros o instituciones oficiales o privadas.

- El género femenino muestra proporcionalmente una mayor producción intelectual, a pesar de que todavía esta relativamente renegado en cuanto a títulos de postgrado obtenidos.

- En la mayoría de los cuatro programas de ciencia y tecnología examinadas existe proporcionalmente una mayor difusión de los resultados de investigación procedente de las universidades privadas.
- No se debe descartar la posibilidad de que los líderes de los grupos de investigación no actualizan las hojas de vida, lo cual tendría como resultado una apreciación errónea de la producción del conocimiento.

- La investigación en los establecimientos de educación superior merece un replanteamiento tanto a nivel interno, como a nivel nacional.

\section{BIBLIOGRAFÍA}

BIOSÍNTESIS. 2001. Actividad investigativa en biodiversidad de Colombia: análisis bibliométrico 1995-1999. Instituto de Investigación de Recursos Biológicos Alexander von Humboldt. Boletín 27. 4p.

CÁRDENAS, F.A. 1999. Doctorados y Sociedades de Conocimiento. Seminario Internacional: la docencia en la universidad contemporánea. Ascun-IcetexSecab. Cali, Agosto 12 y 13.

COLCIENCIAS. 2006a. Directorio de Grupos Colombianos de Investigación Científica y Técnica e Innovación. Programa Nacional de Ciencia y Tecnologías Agropecuarias. Disponible desde Internet en: http://pamplonita.colciencias.gov. co:8081/scienti/htlm/grupos.htlm (con acceso 14-18/11/05 y 16-20/01/06).

COLCIENCIAS. 2006b. Currículo CvLAC. Programa Nacional de Ciencia y Tecnologías Agropecuarias. Disponible desde Internet en: http://pamplonita. colciencias.gov.co:8081/scienti/htlm/curriculums.htlm (con acceso 14-18/11/05 y 1620/01/06).

COLCIENCIAS. 2006c. Directorio de Grupos Colombianos de Investigación Científica y Técnica e Innovación. Programa Nacional de Ciencias del Medio Ambiente y Hábitat. Disponible desde Internet en: http://pamplonita.colciencias.gov. co:8081/scienti/htlm/grupos.htlm (con acceso 14-18/11/05 y 16-20/01/06).

COLCIENCIAS. 2006d. Currículo CvLAC. Programa Nacional de Ciencias del Medio Ambiente y Hábitat. Disponible desde Internet en: http://pamplonita. 
colciencias.gov.co:8081/scienti/htlm/curriculums. htlm (con acceso 14-18/11/05 y 16-20/01/06).

COLCIENCIAS. 2006e. Directorio de Grupos Colombianos de Investigación Científica y Técnica e Innovación. Programa Estudios Científicos de la Educación. Disponible desde Internet en: http:// pamplonita.colciencias.gov.co:8081/scienti/htlm/grupos.htlm (con acceso 26/01/06-03/02/06).

COLCIENCIAS. 2006f. Directorio de Grupos Colombianos de Investigación Científica y Técnica e Innovación. Programa Estudios Científicos de la Educación. Disponible desde Internet en: http:// pamplonita.colciencias.gov.co:8081/scienti/htlm/curriculums.htlm (con acceso 26/01/06-03/02/06).

COLCIENCIAS. 2005. Índice Bibliográfico Nacional-Publindex. Disponible desde Internet en: www.colciencias.gov.co (con acceso 16/11/05).

GONZÁLEZ G., M.I.; PÉREZ S., E. 2002. Ciencia, tecnología y género.Rev. Iberoam. Ciencia, Tecn., Soc. Innov. 2, Enero-Abril 2002. Disponible desde Internet en: http://www.oei.es/revistactsi/numero2/ valenti.htm (con acceso 04/03/07).

ICFES. 2003. La búsqueda de la visibilidad a través de la calidad: reto del editor. Serie Calidad de la Educación Superior No. 9. Bogotá. 145p.

LUCIO, J. 2003. Ciencia y Tecnología en la Universidad Colombiana. Observatorio Colombiano de Ciencia y Tecnología. Bogotá. 65p.

MENDOZA, C.I. 2007. ¿El periodismo se olvidó de la ciencia nacional?. El Tiempo, Sección Información General. Columna de la Defensora. Domingo 4 de 2007. p.24

MURCIA L., D.I. 2004. Investigación educativa. Vestigium 1(1): 54-59.

OBSERVATORIO COLOMBIANO DE CIENCIA Y TECNOLOGÍA (OCyT). 2005. Indicadores de ciencia y tecnología, Colombia 2005. 298p.
OLAYA M., D.L. 2003. La creciente participación de la mujer en la investigación. Colombia: Ciencia y Tecnología 21(1):16-25.

PRESIDENCIA DE LA REPÚBLICA. 2002. Decreto 1279 de Junio 19 de 2002. 30p.

RODRÍGUEZ R., E.A. 2004. Las sociedades del conocimiento y el futuro de la educación en Colombia. Vestigium 1(2):18-23.

ROSSELLI-COCK, D.A. 2005. Enseñar a escribir. Innovación y Ciencia. (A.C.A.C. Colombia). 12(3):1617.

SOLANO-FRANCO, V. 2005. Ver para creer.....y divulgar para creer. Innovación y Ciencia. (A.C.A.C. Colombia). 12(1,2):93-97.

TOVAR ROJAS, P. 2002. Género y Ciencia en Colombia: algunos indicadores. Colombia: Ciencia y Tecnología. 20(2):16-25.

UNAM, MÉXICO. 2006. Doctorado en Ciencias Políticas y Sociales. Disponible desde Internet en: http://www. crim.unam.mx/docencia/Docotrado.htm (con acceso 15/01/06).

UNESCO. 1999. Conferencia mundial sobre la ciencia "La ciencia en el siglo XXI: un nuevo compromiso". Resúmenes. Budapest, 26 de Junio-1 de Julio 1999.

VALENTI LÓPEZ, P. 2002. La sociedad de la información en América Latina y el Caribe: TICs y un nuevo marco institucional. Rev. Iberoam. Cienc., Tecn. Soc. Innov. 2, Abril 2002. Disponible desde Internet en: http://www.oei.es/revistactsi/numero2/valenti.htm (con acceso 04/03/07).

ZENNER DE POLANÍA, I. 2004. El que tiene el saber, tiene el poder. Carta Universitaria. Universidad de Ciencias Aplicadas y Ambientales U.D.C.A, Agosto. p.3.

Recibido: Noviembre 11 de 2006

Aceptado: Marzo 16 de 2007 